\title{
Structure and dynamics of a rotating superfluid Bose-Fermi mixture
}

\author{
Linghua Wen ${ }^{1, *}$ and Jinghong $\mathrm{Li}^{2}$ \\ ${ }^{1}$ College of Science, Yanshan University, Qinhuangdao 066004, China \\ ${ }^{2}$ College of Environment and Chemical Engineering, \\ and College of Science, Yanshan University, Qinhuangdao 066004, China
}

(Dated: July 20, 2018)

\begin{abstract}
We investigate the structure and dynamics of a rotating superfluid Bose-Fermi mixture (SBFM) made of superfluid bosons and two-component (spin up and down) superfluid fermions. A groundstate phase diagram for the nonrotating case of a SBFM with specific parameters is given, where the ground-state configuration of a nonrotating SBFM is mainly determined by the boson-fermion interaction. For the rotating case of a SBFM with a sufficiently large rotation frequency, we show that the system supports a mixed phase and three typical layer separated phases. In particular, the visible vortex formation in the fermionic superfluid exhibits a remarkable hysteresis effect during the dynamical evolution of a rotating SBFM, which is evidently different from the case of rotating two-component Bose-Einstein condensates.
\end{abstract}

PACS numbers: 67.85.Pq, 67.85.Lm, 03.75.Kk, 03.75.Lm

\section{INTRODUCTION}

Superfluidity plays a key role in many fields of physics, such as liquid helium [1], ultracold atomic gases [2, 3], quantum magnets [4], and astrophysics [5]. In particular, superfluid Bose-Fermi mixtures (SBFMs) in ultracold atomic gases have attracted considerable interest recently. It is well known that for liquid helium the superfluidity of bosonic ${ }^{4} \mathrm{He}$ and fermionic ${ }^{3} \mathrm{He}$ can be achieved separately. However, the simultaneous superfluidity for the two isotopes is remarkably prevented by the strong interactions between the two species in spite of such a superfluid ${ }^{4} \mathrm{He}-{ }^{3} \mathrm{He}$ mixture being a long-sought object [6]. By contrast, a superfluid Bose-Fermi mixture (SBFM) in ultracold atomic gases can be realized by using the combination of the Feshbach resonance and radiofrequency techniques. Most recently, a SBFM consisting of condensed ${ }^{7} \mathrm{Li}$ bosons and ultracold ${ }^{6} \mathrm{Li}$ fermions in two-spin states has been produced by Salomon's group [7]. The experimental breakthrough provides new opportunities to study the intriguing properties of SBFMs inaccessible in a Bose-Einstein condensate (BEC) with arbitrary spin [8, 9], pure bosonic superfluid mixtures, and pure fermionic superfluid mixtures. As a matter of fact, many novel physical characteristics have been predicted theoretically in SBFMs, including the saturation effect of nonlinear interaction in the strong-coupling unitarity limit [10], localized Bose-Fermi bright soliton [11], possibility of simulating dense quantum chromodynamics matter 12], phase transition overlapping with phase separation [13], and the Faraday pattern generation [14].

All existing studies of the SBFMs refer to the nonrotating case. Considering that one of the most striking hallmarks of a superfluid is its response to rotation, in this paper we investigate the combined effects

*Electronic address: linghuawen@ysu.edu.cn of rotation and nonlinear interatomic interaction on the exact two-dimensional (2D) topological structure and dynamics of a rotating SBFM, focusing on the superfluid ${ }^{7} \mathrm{Li}-{ }^{6} \mathrm{Li}$ mixture case as the prototype. Here we present a phenomenological dissipation model 15] combining with the unitary Schrödinger equation for an interacting SBFM [10] to describe the dynamics of a rotating SBFM. A ground-state phase diagram for a nonrotating SBFM with specific parameters is given. We find that the ground-state structure of a nonrotating SBFM is mainly determined by the ratio of the boson-fermion (BF) scattering length to the boson-boson (BB) scattering length. Furthermore, it is shown that the rotating SBFM with a sufficiently large rotation frequency can display four steady structures: a mixed phase and three typical layer separated phases. In particular, we show that the generation of visible vortices in the fermionic superfluid displays an evident hysteresis effect during the time evolution of a rotating SBFM. Due to the Cooper pair entity of fermionic superfluid and the presence of $\mathrm{BF}$ and fermion-fermion (FF) interactions, it is demonstrated that the topological structure and the dynamics of a rotating SBFM are evidently different from the usual cases of rotating two-component Bose-Einstein condensates (BECs).

The paper is organized as follows. In Sec. II, we describe a phenomenological model for a rotating SBFM. In Sec. III, we study the equilibrium structure of the rotating SBFM. A phase diagram for a nonrotating SBFM is given, and the phase structures of the rotating SBFMs with various parameter values are analyzed. In Sec. IV, we discuss the dynamics of vortex formation in a rotating SBFM. The conclusion is outlined in the last section.

\section{MODEL}

We consider a superfluid Bose-Fermi mixture composed of superfluid bosons and two-component superfluid 
fermions with equal populations of spin up and down, corresponding to the most favorable condition for Cooper pairing. The superfluid Bose-Fermi gas is confined in a harmonic trap rotating around the $z$ axis with angular velocity $\Omega$. In the present work we use a combined phenomenological model based on a phenomenological dissipation model [15] and a Galilei-invariant nonlinear unitarity model [10] to investigate the dynamics of the vortex formation and the structure of the equilibrium state of a rotating SBFM. In this phenomenological model, the order parameter $\Psi_{b}$ of a bosonic superfluid (i.e., a BoseEinstein condensate) and the order parameter $\Psi_{p}$ of a fermionic superfluid [10] obey the coupled equations in the rotating frame,

$$
\begin{aligned}
(i-\gamma) \hbar \frac{\partial \Psi_{b}}{\partial t}= & {\left[-\frac{\hbar^{2} \nabla^{2}}{2 m_{b}}+U_{b}+\mu_{b}\left(n_{b}, a_{b}\right)-\Omega L_{z}\right] \Psi_{b} } \\
& +G_{b p}\left|\Psi_{p}\right|^{2} \Psi_{b}, \\
(i-\gamma) \hbar \frac{\partial \Psi_{p}}{\partial t}= & {\left[-\frac{\hbar^{2} \nabla^{2}}{2 m_{p}}+U_{p}+\mu_{p}\left(n_{p}, a_{f}\right)-\Omega L_{z}\right] \Psi_{p} } \\
& +G_{b p}\left|\Psi_{b}\right|^{2} \Psi_{p},
\end{aligned}
$$

where for simplicity we assume that the degree of dissipation $\gamma$ of the fermionic superfluid is the same as that of the BEC. $m_{b}$ is the mass of a bosonic atom, $m_{p}=2 m_{f}$ is the mass of a Cooper pair with $m_{f}$ being the mass of a single fermion, and $L_{z}=i \hbar\left(y \partial_{x}-x \partial_{y}\right)$ denotes the $z$ component of the angular-momentum operator. $U_{b}=m_{b}\left(\omega_{b}^{2} r^{2}+\omega_{z b}^{2} z^{2}\right) / 2$ is the external trapping potential for the bosons, $U_{p}=m_{p}\left(\omega_{f}^{2} r^{2}+\omega_{z f}^{2} z^{2}\right) / 2$ is the trapping potential for the fermionic superfluid, and $\omega_{b}\left(\omega_{f}\right)$ and $\omega_{z b}\left(\omega_{z f}\right)$ are the radial trap frequency and the axial trap frequency for the bosons (fermions), respectively. $G_{b p}=4 \pi \hbar^{2} a_{b f} / m_{b f}$, where $a_{b f}$ is the BF $s$-wave scattering length, and $m_{b f}=m_{b} m_{f} /\left(m_{b}+m_{f}\right)$ is the BF reduced mass. The bulk chemical potential of the BEC is given by

$$
\mu_{b}\left(n_{b}, a_{b}\right)=\frac{\hbar^{2}}{m_{b}} n_{b}^{2 / 3} f\left(n_{b}^{1 / 3} a_{b}\right),
$$

where

$$
f(x)=\frac{4 \pi\left(x+\alpha x^{5 / 2}\right)}{1+\lambda x^{3 / 2}+\beta x^{5 / 2}},
$$

$n_{b}$ is the local density of bosons, and $a_{b}$ is the $\mathrm{BB} s$-wave scattering length. Here we choose $(\alpha-\lambda)=32 /(3 \sqrt{\pi})$, $\beta=4 \pi \alpha / \eta$ with $\eta=22.22, \alpha=32 \xi /(3 \sqrt{\pi})$, and $\lambda=$ $32(\xi-1) /(3 \sqrt{\pi})$ with $\xi=1.1[10]$. The bulk chemical potential of the Fermi superfluid is expressed as [10, 16, 17]

$$
\mu_{p}\left(n_{p}, a_{f}\right)=\frac{2 \hbar^{2}}{m_{p}}\left(6 \pi^{2} n_{p}\right)^{2 / 3} g\left(2^{1 / 3} n_{p}^{1 / 3} a_{f}\right),
$$

where

$$
g(x)=1+\frac{\delta x}{1-k x}
$$

$n_{p}=n_{f} / 2$ is the local density of Cooper pairs with $n_{f}$ being the total local density of fermions, $a_{f}$ denotes the attractive $\mathrm{FF}$ scattering length, $\delta=20 \pi /\left(3 \pi^{2}\right)^{2 / 3}$, and $k=\delta /(1-\zeta)$ with $\zeta=0.44$. The choices of the parameters in Eqs. (4) and (6) are consistent with the unitarity and the Lee-Yang-Huang limits [10, 18, 19] as well as the relevant results of the Monte Carlo calculations 20 24].

This phenomenological model is a variation of that in [26, 27] and a generalization of that of a rotating singlecomponent BEC [15], and it has good predictive power [28]. The largest merit of the phenomenological dissipation model is that by virtue of this model one can obtain not only the steady states of a rotating system but also reveal the dynamics of vortex formation. In addition, the present model is valid from weak coupling to unitarity for both bosons and fermions [10].

As a matter of fact, the unitary Schrödinger model for nonrotating superfluid fermions was presented in Refs. [10, 17, 25], where the bulk chemical potential was given by Eqs. (5) and (6) (see also Eqs. (10), (11) and (28) in Ref. [10]). The lowest-order term $g(x)=1$ in Eq. (6) results in the bulk chemical potential of a Fermi superfluid in the absence of FF interaction $\left(a_{f}=0\right)$. Furthermore, the next-order term $g(x)=1+\delta x$ can lead to a known analytical result in the small-gas-parameter regime (medium value FF scattering length $a_{f}$ ) as obtained in Ref. [18]. When $n_{p}^{1 / 3} a_{f}$ tends to negative infinity (the so-called unitary limit), the function $g(x)$ obviously approaches an asymptotic value $(1-\delta / k)$. Thus the function (5) with Eq. (6) provides a smooth interpolation between the bulk chemical potential of a Fermi superfluid in the weak-coupling limit and that in the unitary limit for both the uniform case and the trapped case 10, 17, 18]. It was shown that the results with the choice of the fitting parameters $\delta=20 \pi /\left(3 \pi^{2}\right)^{2 / 3}, k=\delta /(1-\zeta)$, and $\zeta=0.44$ [10] agreed well with the corresponding Monte Carlo data [23, 24]. Therefore, we will use the choice in the present work.

Next, we consider the two-dimensional problem by assuming the translation invariance along the $z$ axis (i.e., $\left.\omega_{z b}=\omega_{z f}=0\right)$, reducing the order parameters as $\Psi_{i}(\vec{r}, t)=\psi_{i}(x, y, t) / \sqrt{R_{z}}(i=b, p)$ with the typical size $R_{z}$ along the $z$ axis. After a straight-forward calculation, we obtain $2 \mathrm{D}$ coupled equations,

$$
\begin{aligned}
(i-\gamma) \hbar \frac{\partial \psi_{b}}{\partial t}= & {\left[-\frac{\hbar^{2}}{2 m_{b}}\left(\nabla_{x}^{2}+\nabla_{y}^{2}\right)+F\left(\psi_{b}\right)-\Omega L_{z}\right] \psi_{b} } \\
& +\left[\frac{m_{b} \omega_{b}^{2}}{2}\left(x^{2}+y^{2}\right)+\frac{G_{b p}}{R_{z}}\left|\psi_{p}\right|^{2}\right] \psi_{b},(7) \\
(i-\gamma) \hbar \frac{\partial \psi_{p}}{\partial t}= & {\left[-\frac{\hbar^{2}}{2 m_{p}}\left(\nabla_{x}^{2}+\nabla_{y}^{2}\right)+J\left(\psi_{b}\right)-\Omega L_{z}\right] \psi_{p} } \\
& +\left[m_{f} \omega_{f}^{2}\left(x^{2}+y^{2}\right)+\frac{G_{b p}}{R_{z}}\left|\psi_{b}\right|^{2}\right] \psi_{p},(8)
\end{aligned}
$$


where

$$
F\left(\psi_{b}\right)=\frac{4 \pi \hbar^{2}\left(a_{b} R_{z}^{-1}\left|\psi_{b}\right|^{2}+\alpha a_{b}^{5 / 2} R_{z}^{-3 / 2}\left|\psi_{b}\right|^{3}\right)}{m_{b}\left(1+\lambda a_{b}^{3 / 2} R_{z}^{-1 / 2}\left|\psi_{b}\right|+\beta a_{b}^{5 / 2} R_{z}^{-5 / 6}\left|\psi_{b}\right|^{5 / 3}\right)},
$$

and

$$
J\left(\psi_{p}\right)=\frac{2 \hbar^{2}}{m_{p}}\left(6 \pi^{2}\right)^{2 / 3}\left|\psi_{p}\right|^{4 / 3}\left(1+\frac{2^{1 / 3} \delta a_{f}\left|\psi_{p}\right|^{2 / 3}}{1-2^{1 / 3} k a_{f}\left|\psi_{p}\right|^{2 / 3}}\right) .
$$

The initial order parameters are normalized as $N_{i}=$ $\iint \psi_{i}(x, y, t=0) d x d y(i=b, p)$ with $N_{b}$ being the initial number of bosons and $N_{p}$ being the initial number of Cooper pairs $\left(N_{p}=N_{f} / 2\right.$ and $N_{f}$ is the total number of fermions). By introducing the notations $\omega_{b f}=\left(\omega_{b}+\omega_{f}\right) / 2, d_{0}=\sqrt{\hbar /\left(2 m_{b f} \omega_{b f}\right)}, x_{0}=x / d_{0}$, $y_{0}=y / d_{0}, t_{0}=t \omega_{b f}$ and $\Omega_{0}=\Omega / \omega_{b f}$, and replacing the wave functions as $\psi_{i} \rightarrow \sqrt{N_{i}} \psi_{i} / d_{0}$, we obtained the rescaled dimensionless $2 \mathrm{D}$ equations for the mixture

$$
\begin{aligned}
(i-\gamma) \frac{\partial \psi_{b}}{\partial t}= & {\left[-\frac{m_{b f}}{m_{b}}\left(\nabla_{x}^{2}+\nabla_{y}^{2}\right)+C_{1}\left|\psi_{p}\right|^{2}-\Omega L_{z}\right] \psi_{b} } \\
& +\frac{4 \pi \hbar}{m_{b} \omega_{b f}} \frac{\left(A_{1}\left|\psi_{b}\right|^{2}+\alpha B_{1}\left|\psi_{b}\right|^{4 / 3}\right)}{1+\lambda D_{1}\left|\psi_{b}\right|+\beta E_{1}\left|\psi_{b}\right|^{5 / 3}} \psi_{b} \\
& +\frac{m_{b} \omega_{b}^{2}}{4 m_{b f} \omega_{b f}^{2}}\left(x^{2}+y^{2}\right) \psi_{b}, \\
(i-\gamma) \frac{\partial \psi_{p}}{\partial t}= & {\left[-\frac{m_{b f}}{m_{p}}\left(\nabla_{x}^{2}+\nabla_{y}^{2}\right)+C_{2}\left|\psi_{b}\right|^{2}-\Omega L_{z}\right] \psi_{p} } \\
& +A_{2}\left|\psi_{p}\right|^{4 / 3}\left(1+\frac{\delta B_{2}\left|\psi_{p}\right|^{2 / 3}}{1-k B_{2}\left|\psi_{p}\right|^{2 / 3}}\right) \psi_{p} \\
& +\frac{m_{f} \omega_{f}^{2}}{2 m_{b f} \omega_{b f}^{2}}\left(x^{2}+y^{2}\right) \psi_{p},
\end{aligned}
$$

where the number subscript 0 is omitted for simplicity. The corresponding coefficients are $A_{1}=a_{b} N_{b} d_{0}^{-2} R_{z}^{-1}$, $B_{1}=a_{b}^{5 / 2} N_{b}^{3 / 2} d_{0}^{-3} R_{z}^{-3 / 2}, C_{1}=8 \pi a_{b f} N_{p} R_{z}^{-1}, D_{1}=$ $a_{b}^{3 / 2} N_{b}^{1 / 2} d_{0}^{-1} R_{z}^{-1 / 2}, E_{1}=a_{b}^{5 / 2} N_{b}^{5 / 6} d_{0}^{-5 / 3} R_{z}^{-5 / 6}, A_{2}=$ $\left(2 \hbar / m_{p} \omega_{b f}\right)\left(6 \pi^{2} N_{p} d_{0}^{-2}\right)^{2 / 3}, B_{2}=\left(2 N_{p} d_{0}^{-2}\right)^{1 / 3} a_{f}$, and $C_{2}=8 \pi a_{b f} N_{b} R_{z}^{-1}$.

In the following, we numerically solve the $2 \mathrm{D}$ coupled equations (11) and (12) which requires an enormous computational effort. The initial ground-state order parameters $\psi_{b}(x, y, t=0)$ and $\psi_{p}(x, y, t=0)$ of the system can be obtained by the imaginary-time propagation method [29 31] based on the Peaceman-Rachford method 32, 33]. Here we consider a superfluid ${ }^{7} \mathrm{Li}^{6}{ }^{6} \mathrm{Li}$ mixture in harmonic traps satisfying $U_{b}(x, y)=m_{b} \omega_{b}^{2}\left(x^{2}+y^{2}\right)=$ $U_{f}(x, y)=m_{f} \omega_{f}^{2}\left(x^{2}+y^{2}\right)$, with $m_{b}$ being the mass of ${ }^{7} \mathrm{Li}$ atom and $m_{f}$ being the mass of the ${ }^{6} \mathrm{Li}$ atom. The parameters are chosen as $\omega_{b}=2 \pi \times 100 \mathrm{~Hz}, R_{z}=10$ $\mu \mathrm{m}$, and $\gamma=0.03$, corresponding to a temperature of about $0.1 T_{c} 34$. Recently, a mixture of ${ }^{7} \mathrm{Li}$ superfluid and ${ }^{6} \mathrm{Li}$ superfluid has been realized by Salomon's group

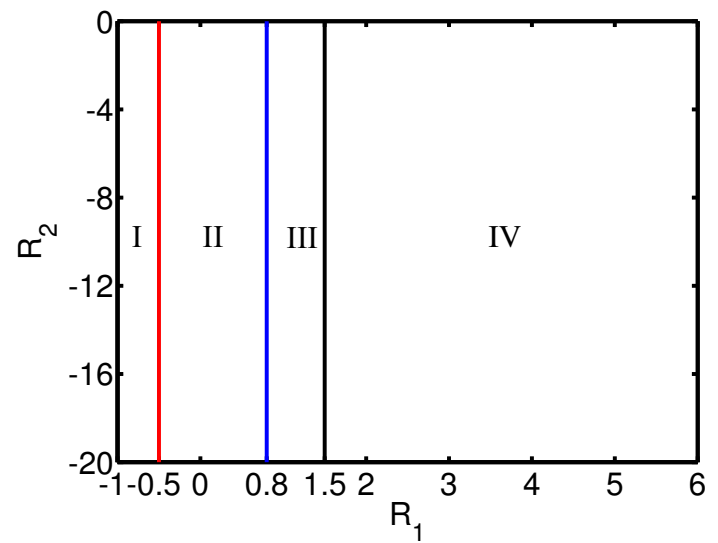

FIG. 1: (Color online) Ground-state phase diagram of a nonrotating superfluid ${ }^{7} \mathrm{Li}-{ }^{6} \mathrm{Li}$ (Bose-Fermi) mixture, where $R_{1}=a_{b f} / a_{b}$ and $R_{2}=a_{f} / a_{b}$. The parameters are $N_{b}=1000$, $N_{p}=100$, and $a_{b}=50 \mathrm{~nm}$. Regions I-IV represent the system collapse regime, mixed phase, layer-separated phase, and inlaid separated phase, respectively.

7]. Thus the above assumption is valid and feasible, and the relevant results can be tested under the current experimental conditions.

\section{STEADY STRUCTURE OF A ROTATING SBFM}

For convenience, we introduce two relative interaction strengths, $R_{1}=a_{b f} / a_{b}$ and $R_{2}=a_{f} / a_{b}$. The FF attractive scattering length $a_{f}$ can be varied from zero to negative infinity, and the absolute value of the BF scattering length $\left|a_{b f}\right|$ is assumed to be not too large (otherwise, it requires special attention [10]).

Figure 1 shows the ground-state phase diagram of a static superfluid ${ }^{7} \mathrm{Li}-{ }^{6} \mathrm{Li}$ mixture with fixed $\mathrm{BB}$ repulsive interaction. The corresponding parameters are $a_{b}=50$ $\mathrm{nm}, N_{b}=1000$, and $N_{p}=100$. There exist four possible phases depending on the values of $R_{1}$ and $R_{2}$, and the typical density profiles corresponding to phases II-IV are displayed in Fig. 2. In Fig. 1, region I denotes the system collapse regime. For a sufficiently strong attractive $\mathrm{BF}$ interaction (here, $R_{1}<-0.5$, i.e., $a_{b f}<-25$ $\mathrm{nm}$ ), the system undergoes a simultaneous collapse of the density profile of the BEC and that of the fermionic superfluid. Physically, the critical value of BF scattering length is governed by the balance between the kinetic energy of bosons and Cooper pairs and the mutual attractive $\mathrm{BF}$ interaction. When the $\mathrm{BF}$ attraction becomes sufficiently strong, it can no longer be stabilized by the kinetic energy. Therefore the mixture lowers its energy via increasing the densities of bosons and Cooper pairs, and finally the bosonic superfluid or the fermionic one or both the bosonic and fermionic superfluids collapse simultaneously due to instability. Region II represents a miscible phase in which the density profile of 
(a1)

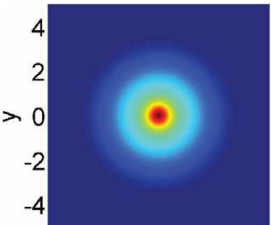

(a2)

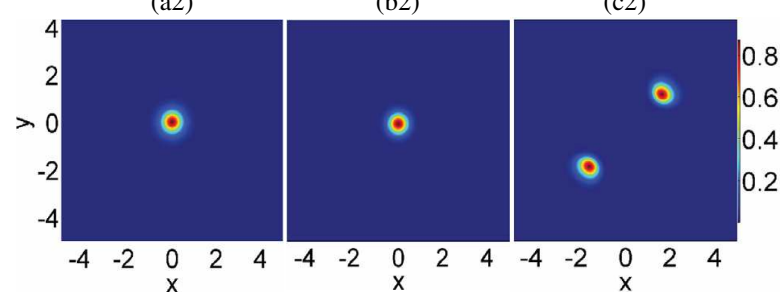

FIG. 2: (Color online) Density profiles (a)-(c) correspond to phases II-IV in Fig. 1, respectively. Here, 1 labels the bosonic superfluid and 2 denotes the fermionic one of the system. (a) $R_{1}=-0.4, R_{2}=-20$, (b) $R_{1}=1, R_{2}=-20$, and (c) $R_{1}=$ $6, R_{2}=-20$. The other parameters are the same as those in Fig. 1. The darker color area indicates the lower density. $x$ and $y$ are in units of $d_{0}$.

the BEC has a much larger spatial extension than that of the fermionic superfluid due to the $\mathrm{BB}$ repulsion and the FF attraction [see Figs. 2(a1) and 2(a2)]. Region III is a layer-separated phase, where the BEC is completely expelled outside the fermionic superfluid because of the strong BF repulsive interaction [Figs. 2(b1) and 2(b2)], which is usually referred as demixing. The similar mixing-demixing transition phenomenon has also been found in the studies of degenerate boson-fermion mixtures 35 39] or two-component BECs [33, 40 43]. Finally, region IV marks an inlaid separated phase in which the fermionic superfluid is divided into two segments and inlaid into the outskirts of the BEC [Figs. 2(c1) and $2(\mathrm{c} 2)]$.

The ground-state structures of SBFMs are different from those of degenerate boson-fermion mixtures [35 39]. In the latter case, there is no $s$-wave interaction between identical fermions in the spin-polarized state due to the Pauli exclusion principle. Depending on the BB and BF interactions, the density profile of a degenerate bosonfermion mixture may display a core-shell-shaped separated phase, where the Fermi gas forms a shell around or a core inside the BEC, or even both [35 39]. However, our simulation shows that in a wide range of parameter values, the SBFM supports neither a separated phase of fermions constituting a shell around the BEC nor a staggered separated phase of fermions becoming both a shell around and a core inside the BEC (see Figs. 1 and 2); even the total number of fermions is much larger than that of bosons. In addition, for a fixed value of $R_{1}$ (i.e., a fixed BF interaction), the variation of the parameter $R_{2}$ (FF interaction) dose not influence the phase structure of the SBFM (Fig. 1). Furthermore, when the repulsive BF interaction of a SBFM is sufficiently strong, the system exhibits an inlaid separated phase, where the fermionic superfluid embeds into the periphery of the bosons by means of two fragment superfluids [Figs. 2(c1) and 2(c2)]. It is known that the fundamental entity of a fermionic superfluid is the Cooper pair [13, 44, 45] and there exists attractive FF interaction between the fermion with spin up and that with spin down. Thus the center-of-mass momentum of each Cooper pair in the fermionic superfluid remains zero, irrespective of the concrete value of $a_{f}$, which may account for the above differences.

On the other hand, the equilibrium properties of the SBFMs are also evidently different from those of twocomponent BECs [33, 40-43]. For the case of twocomponent BECs, by varying the particle numbers of the two BECs or the ratios of intra- and intercomponent interaction strengths, one can obtain many symmetric separated phases [40, 41, 43] and even asymmetric separated phases [33, 42]. Physically, there exist three kinds of actual $s$-wave interactions (including $\mathrm{BB}$ interaction, $\mathrm{BF}$ interaction, and FF interaction) in a SBFM. In particular, when the bosonic and fermionic scattering lengths $a_{b}$ and $\left|a_{f}\right|$ tend to infinity (i.e., in the strong-coupling unitarity limit), both the bosonic and fermionic interactions exhibit unitarity saturation effects due to the constraints of quantum mechanics [10]. In this context, the quasiparticle of a Cooper pair in the fermionic superfluid can just be considered as a "composite boson" rather than a single bosonic atom, which largely results in the differences of the ground-state structures.

In Fig. 3, we display the steady density profiles $\left|\psi_{b}\right|^{2}$ (row 1) and $\left|\psi_{p}\right|^{2}$ (row 2), and the corresponding phase profiles of $\psi_{b}$ (row 3 ) and $\psi_{p}$ (row 4 ) at $t=500$ for a harmonic trap rotating with $\Omega=0.94$. Here the value of the phase varies continuously from 0 to $2 \pi$, and the end point of the boundary between a $2 \pi$ phase line and a 0 phase line denotes a phase defect (i.e., a vortex with anticlockwise rotation). The density profiles of three initial states corresponding to the three columns (left, middle, and right) in Fig. 3 are given in Figs. 2(a)-2(c), respectively.

As shown in Figs. 3(a1) and 3(a3), when the rotating SBFM with an initial state of $R_{1}=-0.4$ and $R_{2}=-20$ reaches an equilibrium state, one can see that there are several visible vortices [15, 28] constituting a triangular lattice in the periphery of the bosonic superfluid. In the meantime, the trap center is occupied by a density peak of bosons due to the attractive BF interaction. However, no visible vortex can be generated in the density of the fermionic superfluid even for large rotation frequency of $\Omega=0.94$ [see Fig. 3(a2)]. From Fig. 3(a4), there exist some phase defects that are located on the outskirts of the superfluid fermions. Since these phase defects are invisible in the in situ density profile of fermionic superfluid and contribute to neither the angular momentum nor the energy of the SBFM, there are referred to as ghost vortices [15, 26 28, 33]. For the case of $R_{1}=1$ and $R_{2}=-20$, there is a large circular density hole of bosons in the trap center which looks like a giant vortex 


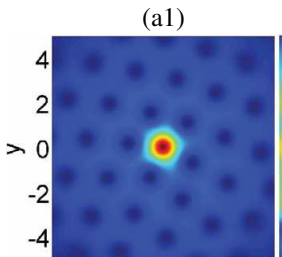

(a2)

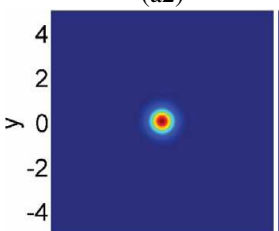

(a3)

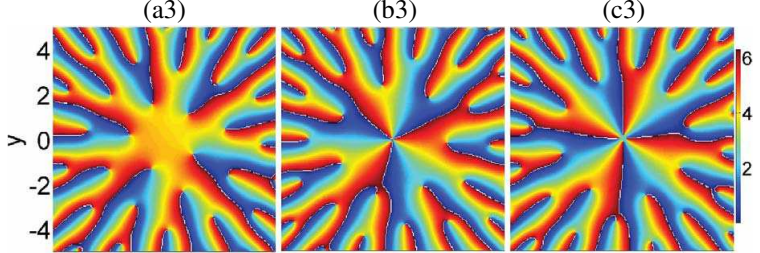

(a4)

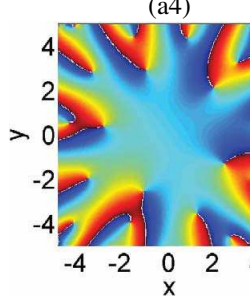

(b1)

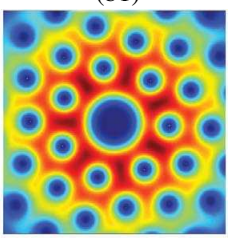

(b2)

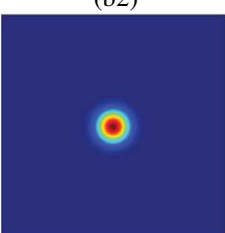

(b3)

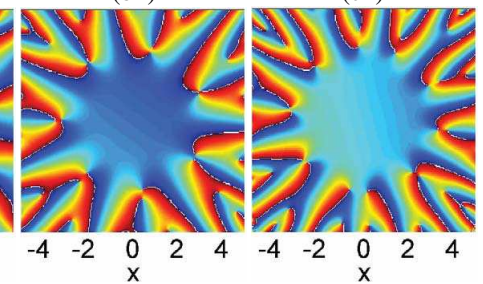

(c1)

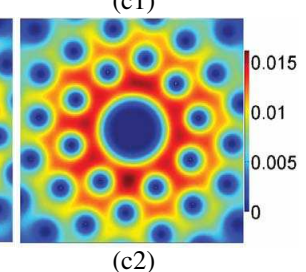

(c2)

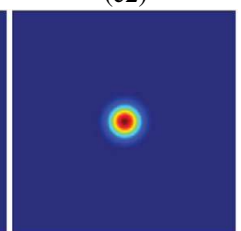

(c3)
FIG. 3: (Color online) Steady density profiles (the top two rows) and phase profiles (the bottom two rows) at $t=500$ after rotating the superfluid Bose-Fermi mixture with $\Omega=$ 0.94, where 1 and 3 denote the bosonic superfluid, while 2 and 4 represent the fermionic superfluid. The initial states corresponding to three cases of $a, b$, and $c$ (from left to right) are illustrated in Figs. 2(a1,a2), Figs. 2(b1,b2), and Figs. $2(\mathrm{c} 1, \mathrm{c} 2)$, respectively. The other parameters are the same as those in Figs. 1 and 2 . The value of phase varies continuously from 0 to $2 \pi$. The darker color area indicates lower density or phase. Here, $x$ and $y$ are in units of $d_{0}$.

(a multiquantized vortex) and is surrounded by an expected visible vortex lattice [Fig. 3(b1)]. In the phase profile displayed in Fig. 3(b3), we find that there are three phase defects in the trap center which are close to each other. The three singly quantized phase defects show that the circular density hole is not a giant vortex. Actually, they are known as singly quantized hidden vortices [15, 28, 33, 46] because they carry significant angular momentum, though they are invisible in the in situ density profile of the BEC. Only after including the hidden vortices can the Feynman rule be satisfied [15, 47, 48]. As seen in Figs. 3(b2) and 3(b4), the fermionic superfluid with ghost vortices distributing on the outskirts of the cloud is completely pulled inside the trap center (i.e., the region of the large density hole of the BEC) due to the competition between the BF repulsion and the rotation repulsion, which indicates a fully separated phase of the rotating SBFM.
With the further increase of the BF repulsive interaction, a nonrotating SBFM will develop into an inlaid separated configuration, where the superfluid fermions lie on the outer edges of the bosons via the form of two fragments [Figs. 2(c1) and 2(c2)]. Counterintuitively, we find that the steady structure of a rotating SBFM with $R_{1}=6$ and $R_{2}=-20$ is similar to that with $R_{1}=1$ and $R_{2}=-20$, as shown in Figs. 3(c1)-3(c4). Here the fermionic superfluid is separately repelled to the trap center region rather than the outskirts of BEC, which is quite different from the nonrotating case. In the presence of dissipation, the steady visible vortex lattice in the BEC is mainly formed by the competition between the rotating driving and the $\mathrm{BB}$ repulsive interaction. The BF repulsion and the centrifugal force acting on the bosons tend to push the fermionic superfluid toward the outside or the inside. Considering as well the coherence of the fermionic superfluid with FF attractive interaction, it evidently prefers to occupy the trap center, especially in the presence of a central density hole for the bosons, because the corresponding potential energy for the superfluid fermions is relatively small such that the system energy of the SBFM reaches the minimum.

In Figs. 4(a1) and 4(a2), we show the density profiles of the BEC and the fermionic superfluid in a static SBFM with $N_{b}=10^{3}, N_{p}=10^{5}, a_{b}=5 \mathrm{~nm}, R_{1}=6$, and $R_{2}=-20$. Compared with the layer-separated phase in Figs. 2(a1) and 2(a2), here the torus density profile of the BEC becomes thinner while the circular density distribution of the fermionic superfluid gets larger. The steady structure at $t=500$ of the SBFM rotating with $\Omega=0.92$ is displayed in Figs. 4(b1) and 4(b2), and the corresponding phase profiles are given in Figs. 4(c1) and 4(c2). From Figs. 4(b1) and 4(c1), we can see that there are no visible vortices, but there exist some hidden vortices in the BEC, where the trap center is occupied by multiple close singly quantized hidden vortices. Here the central blurred region in the phase profile mainly results from the inevitable numerical errors or fluctuations in the numerical computations. In contrast to Fig. 3, a triangular visible vortex lattice made of six visible vortices forms in the rotating fermionic superfluid, as shown in Figs. 4(b2) and 4(c2). Although there is attractive FF interaction between two fermions in a Cooper pair, the fermionic superfluid can display a weak effective repulsive interaction between the Cooper pairs under the appropriate parameters according to Eqs. (21), (5), (6), and (12), which is consistent with the relevant theoretical prediction [49]. This point may explain why visible vortices can be generated in the rotating fermionic superfluid.

\section{DYNAMICS OF VORTEX FORMATION IN A ROTATING SBFM}

In order to reveal the vortex formation process in the rotating superfluid mixture of bosons and Cooper pairs, we consider a bigger superfluid Bose-Fermi system which 
(a1)

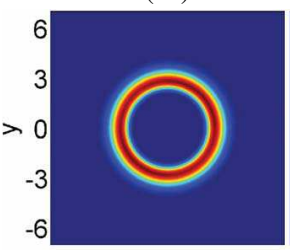

(a2)

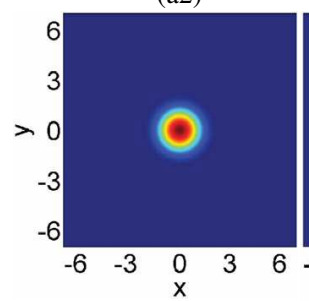

(b1)

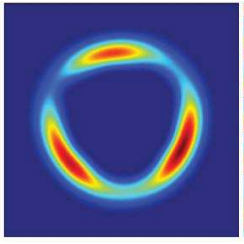

(b2)

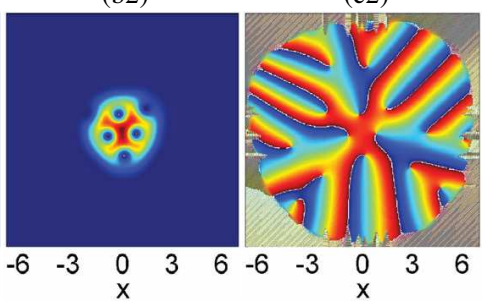

(c1)

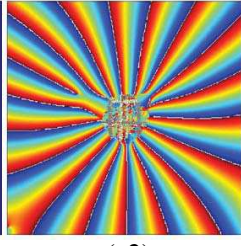

(c2)
FIG. 4: (Color online) (a1), (a2) Ground-state density profiles of a static superfluid ${ }^{7} \mathrm{Li}^{-}{ }^{6} \mathrm{Li}$ mixture with $N_{b}=10^{3}$ and $N_{p}=10^{5}$. (b1), (b2) The steady density profiles at $t=500$ for the superfluid Bose-Fermi system rotating with $\Omega=0.92$. (c1), (c2) The corresponding phase profiles. The relevant parameters are $a_{b}=5 \mathrm{~nm}, R_{1}=6$, and $R_{2}=-20$. Here, 1 denotes the bosonic superfluid, 2 represents the fermionic superfluid, and $x$ and $y$ are in units of $d_{0}$. The darker color area indicates lower density or phase.

requires more computation effort. In Figs. 5(a1) and $5(\mathrm{a} 2)$, we present the ground-state structure of a static SBFM, where the parameters are $N_{b}=10^{4}, N_{p}=10^{5}$, $a_{b}=50 \mathrm{~nm}, R_{1}=6$, and $R_{2}=-0.1$. The steady structure at $t=500$ of the SBFM rotating with $\Omega=0.96$ is shown in Figs. 5(b1) and 5(b2), and the corresponding phase profiles are displayed in Figs. 5(c1) and 5(c2). The initial state of the SBFM at $t=0$ has a shell-shaped separated structure with the fermionic superfluid being surrounded by the bosonic superfluid, which is similar to that in Figs. 2(b1) and 2(b2). In the presence of dissipation, a steady visible vortex lattice forms eventually in the outer of the superfluid BEC [Fig. 5(b1)], where the energy of the rotating SBFM reaches the minimum in the rotating frame. This point is similar to the case of Fig. 3(b1). The large density hole of the bosons corresponds to 22 singly quantized hidden vortices indicated by the phase profile in Fig. 5(c1). The central blurred region in the phase profile is mainly caused by the inevitable numerical errors or fluctuations in the numerical computations. In contrast to the case of $N_{b}=1000$ and $N_{p}=100$ (Figs. 2 and 3), seven evident visible vortices are generated in the fermionic superfluid [see Figs. 5(b2) and $5(\mathrm{c} 2)]$.

The dynamical evolution of the rotating SBFM is illustrated in Fig. 6, where the top two rows denote the time evolutions of the density profiles of the BEC (row 1) and fermionic superfluid (row 2), while the bottom two rows represent those of the phase profiles of the BEC (row 3) and fermionic superfluid (row 4). The evolution times are $t=11.5$ (left), $t=20$ (middle), and $t=60$ (right), respectively. Initially, the superfluid den- (a1)

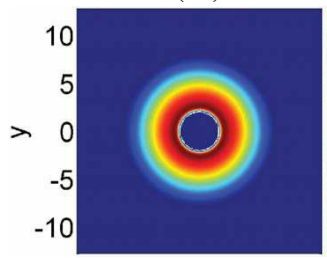

(a2)

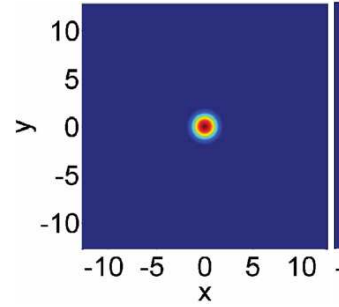

(b1)

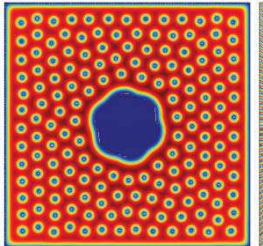

(b2)

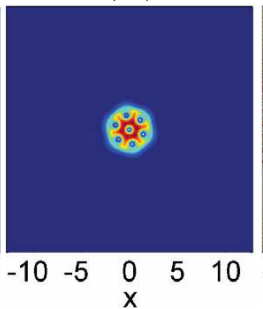

(c1)

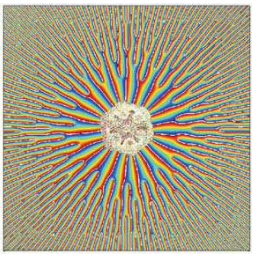

(c2)

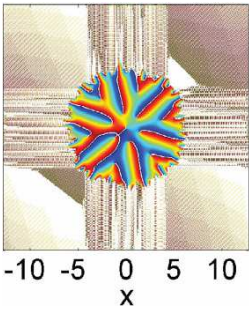

FIG. 5: (Color online) (a1), (a2) Ground-state density profiles of a static superfluid ${ }^{7} \mathrm{Li}-{ }^{6} \mathrm{Li}$ mixture with $N_{b}=10^{4}$ and $N_{p}=10^{5}$. (b1), (b2) The steady density profiles at $t=500$ for the superfluid Bose-Fermi system rotating with $\Omega=0.96$. (c1), (c2) The corresponding phase profiles. The relevant parameters are $a_{b}=50 \mathrm{~nm}, R_{1}=6$, and $R_{2}=-0.1$. Here, 1 denotes the bosonic superfluid, 2 represents the fermionic superfluid, and $x$ and $y$ are in units of $d_{0}$. The darker color area indicates lower density or phase.

sities $\left|\psi_{b}(x, y, t=0)\right|^{2}$ and $\left|\psi_{p}(x, y, t=0)\right|^{2}$ in a stationary isotropic harmonic trap are shown in Figs. 5(a1) and 5(a2). With the development of time, the boundary surface of the bosonic superfluid undergo complex turbulent oscillation and many ghost vortices appear at the outskirts of the BEC, which can be seen in Figs. 6(a1) and 6(a3). In contrast, the fermionic superfluid remains basically unchanged, as shown in Figs. 6(a2) and 6(a4). Essentially, these ghost vortices are generated by collective excitations through the nonlinear atomic interactions and the Landau instability associated with the negative excitation frequency [27, [50] because the rotating harmonic trap is isotropic and has rotation symmetry. This characteristic is evident especially for the component of fermionic superfluid [see Figs. 6(a2)-6(c2) and Figs. 6(a4)-6(c4)]. Thus here the formation mechanism of topological defects is different from the case of a rotating anisotropic harmonic potential, where ghost vortices mainly result from the dynamical instability through the rapid modulation of trapping anisotropy [26, 27, 51, 52]. The angular momentum is transferred into the SBFM via the excitations of surface modes or the generation of visible vortices and hidden vortices. With the further time evolution, some ghost vortices penetrate into the BEC and become visible vortices or hidden vortices due to the Landau instability [see Figs. 6(b1)-6(c1) and Figs. 6(b3)-6(c3)], where the visible vortices arrange themselves irregularly. When the rotating SBFM reaches an equilibrium state, the visible vortices form a triangular lattice such that the energy of the system approaches the minimum in the rotating frame. Compared with the ro- 
(a1)

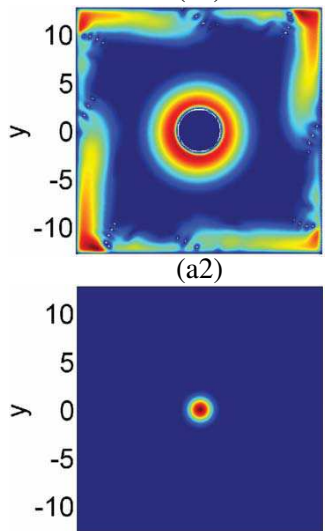

(a3)

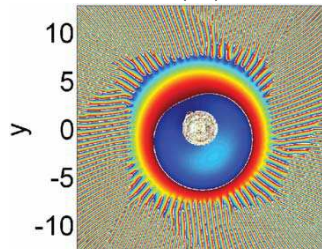

(a4)

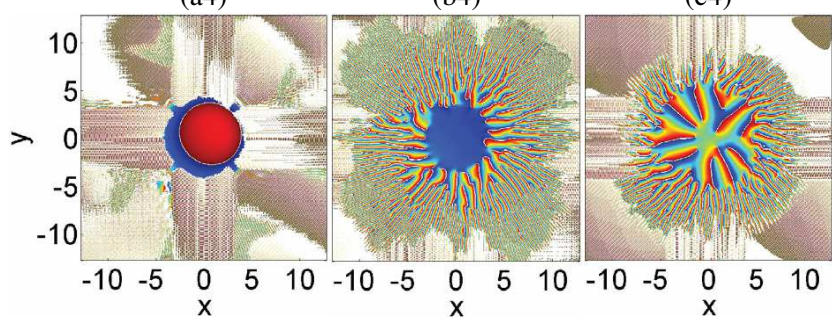

FIG. 6: (Color online) Time evolutions of the density profiles $\left|\psi_{b}\right|^{2}$ (row 1) and $\left|\psi_{p}\right|^{2}$ (row 2) and the corresponding phase profiles (rows 3 and 4) after the superfluid Bose-Fermi system rotates with $\Omega=0.96$, where the other parameters are the same as those in Fig. 5. The value of the phase varies continuously from 0 to $2 \pi$. The time is (a1-a4) $t=11.5$, (b1b4) $t=20$, and (c1-c4) $t=60$. The dark color area indicates the lower density or phase. Here $x$ and $y$ are in units of $d_{0}$, and $t$ is in units of $1 / \omega_{b f}$.

tating BEC, the vortex (including ghost vortex and visible vortex) formation in the rotating fermionic superfluid exhibits an evident hysteresis effect, as shown in Figs. 6 and 5 .

The structure and dynamics of a rotating SBFM are remarkably different from those of rotating two-component BECs. First, in the latter case, the vortices in the two BECs are always generated simultaneously [53 55], while here the vortex formation in the superfluid fermions is far later than that in the $\mathrm{BEC}$ due to the presence of Cooper pair with attractive FF interaction. Second, in the presence of dissipation, there are triangular visible vortex lattices and singly quantized hidden vortices formed in a rotating SBFM. In our simulation, we did not observe the generation of vortex sheet or square vortex lattice occurring in a rotating two-component BEC [53, 55 57]. These structures as well as the giant vortex are suppressed by the dissipation term in our simulation.

\section{CONCLUSION}

In summary, we have studied the structures for rotating and non-rotating superfluid Bose-Fermi mixtures and the dynamics of vortex formation in a rotating superfluid Bose-Fermi mixture. We show that the ratio of BF interaction to $\mathrm{BB}$ interaction plays a key role in determining the ground-state structure of a nonrotating SBFM. For fixed $\mathrm{BB}$ and $\mathrm{BF} s$-wave scattering lengths, the variation of FF (spin-up and -down) $s$-wave scattering length does not influence the phase diagram for a nonrotating SBFM. Furthermore, depending on the choice of parameters, we find that a rotating SBFM with a sufficiently large angular velocity supports four typical steady structures: a mixed phase and three layer-separated phases. In addition, it is shown that different separated phases at the initial time may result in almost the same steady structures. In particular, we find that the generation of visible vortices in the fermionic superfluid exhibits an evident hysteresis effect during the time evolution of a rotating SBFM. Our results indicate that the topological structure and the dynamics of a rotating SBFM are remarkably different from the cases of rotating twocomponent BECs. We expect that our findings can be observed and tested in future experiments. In the mean time, the present investigation provides a way to further test the validity of the unitarity model [10] for a SBFM.

\section{Acknowledgments}

We thank Biao Wu, Yongping Zhang, Li Mao and Yong $\mathrm{Xu}$ for helpful discussions. L.W. acknowledges the research group of Professor Chuanwei Zhang at The University of Texas at Dallas, where part of the computations were carried out. This work was supported by the National Natural Science Foundation of China (Grants No. 11475144, No. 11047033, and No. 11304270), and the Ph.D. Foundation of Yanshan University (No. B846).
[1] A. J. Leggett, Quantum Liquids (Oxford University Press, Oxford, 2006).

[2] L. Pitaevskii and S. Stringari, Bose-Einstein Condensation (Oxford University Press, Oxford, 2003).
[3] C. J. Pethick and H. Smith, Bose-Einstein Condensation in Dilute Gases, 2nd ed. (Cambridge University Press, Cambridge, 2008).

[4] V. Zapf, M. Jaime, and C. D. Batista, Rev. Mod. Phys. 
86, 563 (2014).

[5] S. Liberati and L. Maccione, Phys. Rev. Lett. 112, 151301 (2014).

[6] J. Rysti, J. Tuoriniemi, and A. Salmela, Phys. Rev. B 85, 134529 (2012).

[7] I. Ferrier-Barbut, M. Delehaye, S. Laurent, A. T. Grier, M. Pierce, B. S. Rem, F. Chevy, and C. Salomon, Science 345, 1035 (2014).

[8] T.-L. Ho and L. Yin, Phys. Rev. Lett. 84, 2302 (2000).

[9] Y. Wu, X. Yang, and C. Sun, Phys. Rev. A 62, 063603 (2000).

[10] S. K. Adhikari and L. Salasnich, Phys. Rev. A 78, 043616 (2008).

[11] S. K. Adhikari and L. Salasnich, Phys. Rev. A 76, 023612 (2007).

[12] K. Maeda, G. Baym, and T. Hatsuda, Phys. Rev. Lett. 103, 085301 (2009).

[13] B. Ramachandhran, S. G. Bhongale, and H. Pu, Phys. Rev. A 83, 033607 (2011).

[14] F. Kh. Abdullaev, M. Ögren, and M. P. Sorensen, Phys. Rev. A 87, 023616 (2013).

[15] L. H. Wen, H. W. Xiong, and B. Wu, Phys. Rev. A 82, 053627 (2010).

[16] A. Recati, I. Carusotto, C. Lobo, and S. Stringari, Phys. Rev. Lett. 97, 190403 (2006).

[17] S. K. Adhikari, Phys. Rev. A 77, 045602 (2008).

[18] T. D. Lee and C. N. Yang, Phys. Rev. 105, 1119 (1957).

[19] T. D. Lee, K. Huang, and C. N. Yang, Phys. Rev. 106, 1135 (1957).

[20] D. Blume and C. H. Greene, Phys. Rev. A 63, 063601 (2001).

[21] J. Carlson, S.-Y. Chang, V. R. Pandharipande, and K. E. Schmidt, Phys. Rev. Lett. 91, 050401 (2003).

[22] G. E. Astrakharchik, J. Boronat, J. Casulleras, and S. Giorgini, Phys. Rev. Lett. 93, 200404 (2004).

[23] D. Blume, J. von Stecher, and C. H. Greene, Phys. Rev. Lett. 99, 233201 (2007).

[24] S. Y. Chang and G. F. Bertsch, Phys. Rev. A 76, 021603(R) (2007).

[25] L. Salasnich, N. Manini, and F. Toigo, Phys. Rev. A 77, 043609 (2008).

[26] M. Tsubota, K. Kasamatsu, and M. Ueda, Phys. Rev. A 65, 023603 (2002).

[27] K. Kasamatsu, M. Tsubota, and M. Ueda, Phys. Rev. A 67, 033610 (2003).

[28] L. H. Wen and X. B. Luo, Laser Phys. Lett. 9, 618 (2012).

[29] Y. Zhang, L. Mao, C. Zhang, Phys. Rev. Lett. 108, 035302 (2012).

[30] Y. Xu, Y. Zhang, B. Wu, Phys. Rev. A 87, 013614 (2013).

[31] L. H. Wen, J. S. Wang, J. Feng, and H. Q. Hu, J. Phys.
B 41, 135301 (2008).

[32] D. W. Peaceman and H. H. Rachford, Jr., J. Soc. Ind. Appl. Math. 3, 28 (1955).

[33] L. H. Wen, Y. J. Qiao, Y. Xu, and L. Mao, Phys. Rev. A 87, 033604 (2013).

[34] S. Choi, S. A. Morgan, and K. Burnett, Phys. Rev. A 57, 4057 (1998).

[35] K. Molmer, Phys. Rev. Lett. 80, 1804 (1998).

[36] R. Roth, Phys. Rev. A 66, 013614 (2002).

[37] P. Capuzzi, A. Minguzzi, and M. P. Tosi, Phys. Rev. A 68, 033605 (2003).

[38] S. K. Adhikari and L. Salasnich, Phys. Rev. A 75, 053603 (2007).

[39] L. H. Wen, Y. P. Zhang, and J. Feng, J. Phys. B 43, $225302(2010)$.

[40] T.-L. Ho and V. B. Shenoy, Phys. Rev. Lett. 77, 3276 (1996).

[41] H. Pu and N. P. Bigelow, Phys. Rev. Lett. 80, 1130 (1998).

[42] M. Trippenbach, K. Goral, K. Rzazewski, B. Malomed, and Y. B. Band, J. Phys. B 33, 4017 (2000).

[43] L. Wen, W. M. Liu, Y. Cai, J. M. Zhang, and J. Hu, Phys. Rev. A 85, 043602 (2012).

[44] S. K. Adhikari and L. Salasnich, New J. Phys. 11, 023011 (2009).

[45] K. B. Gubbels and H. T. C. Stoof, Phys. Rev. A 84, 013610 (2011).

[46] T. Mithun, K. Porsezian, and B. Dey, Phys. Rev. A 89, 053625 (2014).

[47] A. L. Fetter, Rev. Mod. Phys. 81, 647 (2009).

[48] N. R. Cooper, Adv. Phys. 57, 539 (2008).

[49] P. Pieri and G. C. Strinati, Phys. Rev. Lett. 91, 030401 (2003).

[50] B. Wu and Q. Niu, Phys. Rev. A 64, 061603(R) (2001).

[51] F. Dalfovo and S. Stringari, Phys. Rev. A 63, 011601(R) (2000).

[52] K.W. Madison, F. Chevy, V. Bretin, and J. Dalibard, Phys. Rev. Lett. 86, 4443 (2001).

[53] K. Kasamatsu, M. Tsubota, and M. Ueda, Phys. Rev. Lett. 91, 150406 (2003).

[54] S. J. Woo, S. Choi, L. O. Baksmaty, and N. P. Bigelow, Phys. Rev. A 75, 031604 (2007).

[55] P. Mason and A. Aftalion, Phys. Rev. A 84, 033611 (2011).

[56] P. Kuopanportti, J. A. M. Huhtamäki, and M. Möttönen, Phys. Rev. A 85, 043613 (2012).

[57] N. Ghazanfari, A. Keleş, and M. Ö. Oktel, Phys. Rev. A 89, 025601 (2014). 